\title{
Steigende Grundwasserspiegel als Herausforderung für zukünftiges Grundwassermanagement in alpinen Tälern
}

\author{
Timo Kessler ${ }^{1,2}$ (D) $\cdot$ Robert Marschallinger ${ }^{3}$ - Giorgio Höfer-Öllinger ${ }^{4}$ \\ Eingegangen: 2. Februar 2021 / Überarbeitet: 19. Mai 2021 / Angenommen: 26. Mai 2021 / Online publiziert: 2. November 2021 \\ (c) Der/die Autor(en) 2021
}

\section{Zusammenfassung}

In einigen alpinen Gemeinden in Österreich sind die lokalen Grundwasserspiegel in den letzten Jahrzehnten auf kritische Niveaus angestiegen. Einerseits werden die Flächenversiegelung, der Wegfall von Retentionsräumen und die lokale Versickerung von Niederschlagswasser als Gründe für diese Entwicklung gesehen. Andererseits unterliegen Grundwasserressourcen dem Klimawandel, der sich örtlich mit variabler Grundwasserneubildung durch extreme Niederschlagsereignisse oder starke Schneeschmelze bemerkbar macht. In diesem Beitrag wird anhand einer Modellierungsstudie die Sensitivität eines lokalen, oberflächennahen Grundwasserleiters in Bezug auf naturräumliche, klimatische und anthropogene Entwicklungen analysiert. Es zeigt sich, dass eine unkontrollierte Interaktion von Oberflächengewässern maßgeblich und langfristig in den Grundwasserhaushalt eingreifen kann. Dies gilt insbesondere, wenn die Transferrate von Oberflächengewässern durch hydraulische Maßnahmen (Drainagen, Dichtwände, Sohlabdichtungen) oder natürliche Phänomene wie Hochwasser verändert wird. Die Studie verfolgt das Ziel, multiple Einflussfaktoren auf alpines Grundwassermanagement zu untersuchen und im Hinblick auf mögliche zukünftige Entwicklungen zu bewerten.

Schlüsselwörter Grundwasseranstieg $\cdot$ Neubildung $\cdot$ Flächenversiegelung $\cdot$ Klimawandel $\cdot$ Alpine Täler

\section{Rising groundwater levels is challenging future groundwater management in alpine valleys}

\begin{abstract}
In some Alpine communities in Austria, local groundwater levels have risen to critical levels. Increasing land sealing, loss of retention areas and local infiltration of precipitation are seen as reasons for this development. Furthermore, groundwater resources are subject to climate change, which is locally perceptible by variable groundwater recharge through extreme precipitation events or snow melt. In this paper, a modelling study is employed to analyse the sensitivity of a local, near-surface groundwater aquifer with regard to physiographical, climatic and anthropogenic developments. It is shown that an uncontrolled interaction with surface waters can significantly affect the groundwater balance, for example if the groundwater/surface water exchange rate is altered by hydraulic measures (e.g. drainage systems, barrier walls) or by natural phenomena such as flooding events. This study aims to assess multiple influences on alpine groundwater management and to evaluate their role for potential future developments.
\end{abstract}

Keywords Groundwater rise $\cdot$ Recharge $\cdot$ Area sealing $\cdot$ Climate trends $\cdot$ Alpine valleys

Timo Kessler

timo.kessler@uni-greifswald.de

Robert Marschallinger

office@marschallinger.eu

Giorgio Höfer-Öllinger

giorgo.hoefer-oellinger@geoconsult.com

1 Georesearch Forschungsgesellschaft mbH, Urstein Süd 13, 5412 Puch bei Hallein, Österreich
2 Institut für Geographie und Geologie, Universität Greifswald, Friedr.-Ludwig-Jahn-Str. 17A, 17487 Greifswald, Deutschland

3 Marschallinger Geoinformatik, Fischtagging 87, 5201 Seekirchen, Österreich

4 Geoconsult ZT GmbH, Ustein Süd 13, 5412 Puch bei Hallein, Österreich 


\section{Einleitung}

Die Nutzung alpiner Täler durch den Menschen basiert auf einer Entwicklung über Jahrtausende. Siedlungsdruck und bauliche Maßnahmen zur Abwehr von Naturgefahren wie beispielsweise Hochwasser oder Muren haben die naturräumlichen Rahmenbedingungen gravierend verändert. Tiefbauten, Entwässerungsbauwerke, Flutungen im Hochwasserfall oder punktuelle Versickerungen von Niederschlagswasser beeinflussen das hydrologische Regime und steigern die Systemkomplexität. Daneben unterliegen Grund- und Oberflächenwasserressourcen den Veränderungen des Klimawandels inklusive einer steigenden Variabilität der Grundwasserneubildung aufgrund von extremen Niederschlagsereignissen oder starker Schneeschmelze (Eckhardt und Ulbrich 2003; Stocker et al. 2013; Klammler et al. 2013). In Österreich werden steigende Grundwasserspiegel seit den 1980er-Jahren beobachtet, was nicht per se auf den Klimawandel, sondern auch auf veränderte anthropogene Nutzungen zurückzuführen ist (Haas und Birk 2020).

In dieser Studie wird untersucht, inwieweit klimatische, naturräumliche und anthropogene Faktoren zu Schwankungen des Grundwasserstands in alpinen Aquiferen führen und welche Art von Wechselwirkungen auftreten können. Taylor et al. (2013) beschreiben, dass ein veränderliches Klima gravierende Auswirkungen auf die Grundwasserdynamik von alpinen Aquiferen haben kann, da gerade im Alpenraum die Klimaerwärmung stark ausgeprägt ist (Gobiet et al. 2014; Haas und Birk 2019). Insbesondere in Trockenperioden oder während Hitzewellen reagieren oberflächennahe oder räumlich eng begrenzte Grundwasserleiter sehr sensibel (Bender et al. 2021). Die Grundwasserneubildung von Talaquiferen hängt unter anderem von Niederschlag, Evapotranspiration, Schneeakkumulation und Schneeschmelze in den topographisch höher gelegenen Einzugsgebieten ab (Klammler et al. 2013; Kløve et al. 2014; Blöschl et al. 2018). Gleichwohl ist die Interpretation von Grundwasserspiegelschwankungen basierend auf klimatischen Veränderungen aufgrund von zeitlichen Verzögerungen (Bender et al. 2021) sowie der Skalierung von meteorologischen Daten mit großen Unsicherheiten behaftet (Scibek und Allen 2006). In Österreich sind die Niederschlagssummen im Norden und inneralpin seit 1975 zwischen 10 und $15 \%$ gestiegen und lassen dort für die Zukunft eine Zunahme der Grundwasserneubildung im Winter und Frühjahr erwarten (Blöschl et al. 2011, 2018). Dieser Zusammenhang wird in Haas und Birk (2019) für den Zeitraum seit 1980 durch die Anwendung des Standardized Groundwater level Index SGI (Bloomfield und Marchant 2013) bestätigt.

Verkarstete Gebirgseinzugsgebiete, wie sie im Alpenraum häufig vorkommen, reagieren besonders sensitiv auf hydraulische Impulse (Finger et al. 2013; Chen et al. 2018). Freigesetztes Wasser aus Starkniederschlägen oder Schneeschmelze versickert rasch und infiltriert in tiefer liegende Aquifere. Dabei ist die hydraulische Durchlässigkeit ein maßgeblicher Parameter ob Grundwasserkörper von klimatischen Veränderungen unmittelbar oder zeitlich verzögert betroffen sind (Tague und Grant 2009). Ähnlich argumentieren Arnoux et al. (2021) indem sie die Beschaffenheit eines Grundwasserkörpers als maßgeblichen Parameter für die Veränderlichkeit beschreiben. Sie zeigen, dass unter anderem das Speichervermögen eines Grundwasserleiters Einfluss darauf hat, wie ein alpiner Aquifer auf ein verändertes Klima reagiert. Ausschlaggebend ist demnach der Anteil quartärer Sedimente, welche variable hydraulische Durchlässigkeiten aufweisen und Wasser über einen längeren Zeitraum speichern und abgeben können (Arnoux et al. 2020). Bonat et al. (2020) weisen in diesem Zusammenhang darauf hin, dass das Zusammenwirken von Neubildung (vorrangig durch Kluftgrundwasserleiter) und Speicherung (in Porengrundwasserleitern) die Grundwasserdynamik bestimmt.

Die Interaktion von Grund- und Oberflächengewässern ist ebenfalls entscheidend für die zeitliche Variabilität des Grundwasserstandes. Diese hängt vom Wasserstand der Fließgewässer $a b$ und variiert sowohl räumlich als auch zeitlich stark (Kløve et al. 2014; Blumstock et al. 2016). In den vergangenen 30 Jahren hat in Österreich in kleinen Einzugsgebieten nördlich des Alpenhauptkammes die Hochwasserhäufigkeit signifikant zugenommen (Blöschl et al. 2011, 2018; Haas und Birk 2019). Gleichzeitig haben im selben Zeitraum auch die Niederwasserabflüsse in $14 \%$ aller Einzugsgebiete zugenommen (Blöschl et al. 2011). Eine veränderte Interaktion und Infiltration der Grundwasserkörper erscheint daher zumindest wahrscheinlich.

Während in ländlichen oder Gebirgsregionen die geoklimatischen Einflüsse auf das Grundwasser ausschlaggebend sind, dominieren in urbanen Räumen anthropogene Einflussgrößen (Epting et al. 2021). Zu den sozioökonomischen Szenarien zählen in erster Linie Veränderungen der Landnutzungstypen (Holman 2006; Barua et al. 2021) oder die Bewässerung von landwirtschaftlichen Flächen (Lasagna et al. 2020). Lokale Effekte durch Flächenversiegelung, dezentrale Regenwasserversickerungen oder hydraulische Verbauungen, wie sie etwa für die Energiegewinnung, den Hochwasserschutz oder zur Abwehr von Naturgefahren errichtet werden, sind dagegen wenig untersucht und dokumentiert.

Für die Untersuchung der oben beschriebenen, multiplen Einflussfaktoren auf alpine Grundwasserkörper wurde ein kleinräumiges Einzugsgebiet im Umfeld der Gemeinde Rif im Salzachtal ausgewählt. Hier existiert eine Anzahl von Zeitreihen des Grundwasserstandes über einen Zeitraum von 25 Jahren. In drei Messstellen (Au B134, Taxach Br13, 


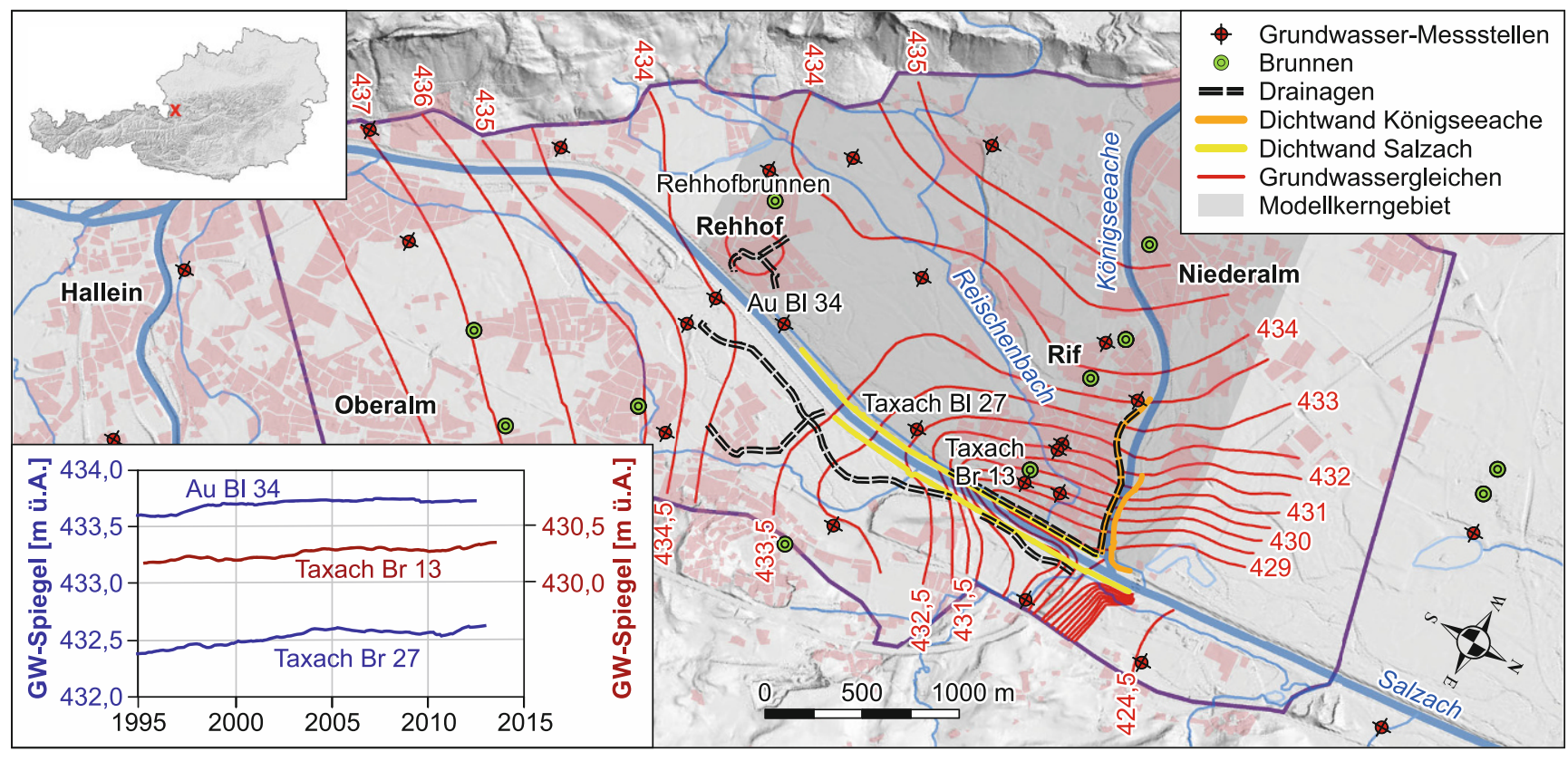

Abb. 1 Untersuchungsgebiet mit Modellkerngebiet Rehhof/Rif/Niederalm (grau unterlegter Bereich) sowie interpolierter Isohypsen und hydraulisch relevanter Einbauten wie Drainagen und Dichtwänden. In der Teilabbildung links unten sind die Zeitreihen von drei salzachnahen Grundwassermessstellen (AuB134, Taxach B127 und Taxach Br13) dargestellt

Fig. 1 Study area with area of interest Rehhof/Rif/Niederalm (highlighted area) with groundwater contour lines and hydraulically relevant installations such as drainages and sealing walls. In the lower left inset, the time series of three near-Salzach monitoring wells (AuB134, Taxach B127 und Taxach Br13) are shown

Taxach B127) ist ein kontinuierlicher Grundwasseranstieg von 20 bis $30 \mathrm{~cm}$ im Vergleich zum Ausgangsjahr 1995 dokumentiert (Abb. 1). Rif erfuhr in diesen Jahren einen steigenden Siedlungsdruck, welcher sich in einer raschen Neubebauung inklusive Flächenversiegelung, Tiefbau und punktuellen Regenwasserversickerungen bemerkbar macht.

Mithilfe eines Grundwasserströmungsmodells wurde das Fließverhalten unter Einbezug aller hydraulisch relevanten $\mathrm{Zu}$ - und Abströme sowie technischer Einbauten abgebildet. Ausgehend von einem kalibrierten Referenzmodell wurde die Sensitivität des Grundwasserstands im Hinblick auf räumliche Parameter wie Änderung der Flächennutzung, bauliche Maßnahmen, Interaktion von Grund- und Oberflächengewässern, sowie numerische Randbedingungen getestet. Daneben wurden die Auswirkungen eines veränderten Niederschlagseintrags für das Land Salzburg (Chimani et al. 2016) für die Zeiträume bis 2050 bzw. 2100 berechnet. Das Modell liefert quantitative Aussagen über das $\mathrm{Zu}$ sammenwirken der Einflussgrößen und gibt möglicherweise Hinweise auf die Ursachen der beobachteten zeitlichen Entwicklung des Grundwasserstands im Untersuchungsgebiet.

Die Studie kann darüber hinaus grundwasserhydraulische Beurteilungskriterien liefern, die für die Erstellung von Richtlinien für das Grundwassermanagement alpiner Städte und Gemeinden hilfreich sein können. Dieses muss der Dynamik vorhandener Grundwasserressourcen Rechnung tragen, um sie nachhaltig zu nutzen und für die Zukunft sichern zu können. Insbesondere, da das Zusammenspiel aus Klimawandel, veränderter Landnutzung und Wasserwirtschaft negative Auswirkungen sowohl für die Nutzung als auch für die Neubildung der Grundwasserkörper haben kann (Haas und Birk 2019).

\section{Untersuchungsgebiet}

Das Untersuchungsgebiet befindet sich etwa $10 \mathrm{~km}$ südlich der Stadt Salzburg am Rand der nördlichen Kalkalpen (Abb. 1). Es umfasst das Salzachtal zwischen den Gemeinden Niederalm im Norden und Oberalm im Süden mit einer Nord-Süd-Ausdehnung von $5,5 \mathrm{~km}$. Im Westen und Osten wird das Gebiet mit einer Breite von etwa $2,5 \mathrm{~km}$ von den Gebirgsmassiven des Hohen Göll bzw. der Osterhorngruppe begrenzt. Der Fokus der Modellierung befindet sich im Ortsgebiet der Gemeinde Rif und Niederalm entlang der Königseeache sowie südlich davon (schraffierter Bereich in Abb. 1). Die südliche Begrenzung des Modellkerngebietes befindet sich etwa auf Höhe des Rehhofbrunnens. Die Modellraumerweiterung im Norden, Osten und Süden wurde für die Kalibrierung des Modells und die Definition von Randbedingungen notwendig.

Hydraulisch wird das Untersuchungsgebiet von den Oberflächengewässern Salzach und Königseeache sowie den daran gekoppelten Grundwasserströmen kontrolliert. 
Der Hauptgrundwasserstrom fließt von Süd nach Nord und vereinigt sich im Modellgebiet mit dem Grundwasserkörper der Königseeache, welcher aus westlicher Richtung der Salzach zufließt. Stichtagsmessungen aus dem Frühjahr 2015 und daraus interpolierte Grundwassergleichen (Abb. 1) zeigen, dass im Bereich der Rehhofsiedlung eine Unterströmung der Salzach besteht und weiter nördlich sehr flache hydraulische Gradienten herrschen. Die Drainagen entlang Königseeache und Salzach ziehen Wasser nach Norden $a b$, gleichzeitig entwässert die Rehhof-Drainage in diesem Bereich nach Süden und wirkt dieser Strömungsrichtung entgegen. Aus Untersuchungen ist ebenfalls bekannt, dass die Königseeache im Bereich Niederalm Seihwasser in den Grundwasserkörper infiltriert (Geoconsult 2017). Direkt unterstrom des Zusammenflusses von Salzach und Königseeache befindet sich das Kraftwerk Urstein, welches durch Stauhöhe, Staulegungen, sowie bauliche Maßnahmen das Grundwassergeschehen beeinflusst und steile hydraulische Gradienten begünstigt (Kersch 2016; Geoconsult 2017). Der Rückstau des Kraftwerks betrifft die letzten $900 \mathrm{~m}$ Fließstrecke der Königseeache sowie den gesamten Verlauf der Salzach im Modellgebiet. Uferdrainagen befinden sich am südlichen Ufer der Königseeache sowie beidseitig der Salzach. Diese ziehen infiltrierendes Wasser der Königseeache in Richtung der Salzach ab und stabilisieren das Grundwasserniveau. Im Untersuchungsgebiet existieren einige Brunnen (Abb. 1), von denen die Mehrzahl für den Betrieb von Grundwasserwärmepumpen genutzt werden und daher in der Bilanz für das Grundwassermanagement keine Relevanz haben. Der Rehhofbrunnen wird zur lokalen Trinkwasserversorgung genutzt, zeigt aber über den Beobachtungszeitraum keine gravierenden Entnahmeschwankungen.

Der geologische Aufbau des Untersuchungsgebietes besteht im Wesentlichen aus fluvialen Schüttungen von Salzach und Königseeache, welche durch Stillwassersedimente (Feinsand und Schluff des "Salzburger Seetons") unterlagert sind (Stummer 1947; Seefeldner 1954; Brandecker 1974; van Husen 1979; Donadel et al. 2014; Pomper et al. 2017). Letztere bilden eine hydraulische Barriere im Liegenden. Der Grundwasserleiter ist aus mittelbis feinkörnigen Kiesen und Sand zusammengesetzt, die zudem oberflächennah linsenförmige Einschaltungen von Hochflutsanden aufweisen. Komplexe Ablagerungsverhältnisse bestehen im südlichen Modellgebiet, wo sich Ausläufer des Deltas der Alm in Kiesfazies bis zum Rehhofbrunnen erstrecken, distal - gegen Nord - zunehmend versanden bzw. im Bottom Set auskeilen. Da das eigentliche Delta der Königseeache im Salzburger Becken am Nordfuß des Untersberges liegt (Czernin-Chudenitz et al. 1978; Wessely et al. 2016) und der Durchbruch gegen Ost ins Salzachtal in der Sedimentationsfolge sehr spät erfolgen musste (Donadel et al. 2014), ist für deren letzten Flussab- schnitt im Salzachtal die Geologie eines verzweigten Flusssystems anzunehmen. Ein typisches dreidimensionales Delta konnte sich hier nicht ausprägen. An den Talrändern umrahmt Festgestein der benachbarten Kalkalpenmassive das Becken (Plöchinger 1983, 1987; Braunstingl und Pestal 2005). Hier werden dem Aquifer geringfügige Zuflüsse aus Hangwässern und Kluft- bzw. Karstgrundwasser zugeführt. Eine direkte Anbindung des Karst- und Porengrundwasserleiters mit mengenmäßig relevantem Wasseraustausch kann jedoch ausgeschlossen werden (Höfer-Öllinger 2020). Der wesentliche $\mathrm{Zu}-$ bzw. Abfluss verteilt sich auf den regionalen Grundwasserfluss entlang der Achse des Salzachtales sowie den Grundwasserzustrom der Königseeache.

\section{Methodik}

Für die Sensitivitätsuntersuchung des Grundwasserkörpers wurde ein numerisches Finite-Elemente-Strömungsmodell mit der Software Feflow (Diersch 2014) erstellt. Ein dreidimensionaler Modellaufbau wurde bevorzugt, um die unterschiedlich durchlässigen Sedimentklassen und Feinkorneinschaltungen innerhalb der fluvialen Lockergesteinshorizonte von Salzach und Königseeache abbilden zu können. Die lithologische Sedimentverteilung wurde mit stochastischer multiple-point Simulationen erstellt und in das Modell integriert. Die Berechnung der Potenzialverteilung erfolgte stationär. Für eine Korrelation der Oberflächengewässerpegel mit dem Grundwasserstand wurde eine instationäre Berechnung in Betracht gezogen, im Verlauf der Modellierungsarbeiten jedoch verworfen. Die Messintervalle der verfügbaren Zeitreihen unterscheiden sich stark, sodass Abflussspitzen oder Niedrigwasserperioden nur unzureichend korreliert werden konnten.

\section{Randbedingungen}

Die Modellränder im Norden und Süden wurden mit Festpotenzialen definiert. Der Wasseraustausch von Grundund Oberflächenwasser wurde mit Transfer-Randbedingungen (Leaky RB) berücksichtigt. Diese definieren die In-/ Exfiltration basierend auf der hydraulischen Durchlässigkeit und der Mächtigkeit der kolmatierten Berandung und Sohlschicht der Flussbetten. Die hydraulische Durchlässigkeit liegt typischerweise 2-3 Größenordnungen niedriger als die der umgebenden Matrix (Schälchli 1993; Fette et al. 2004). Die Festgesteinsränder infiltrieren örtlich geringe Mengen an Bergwasser. Diese Zuflüsse wurden in Harum et al. (2016) untersucht und bilden die Basis für die Definition der Flussraten (Neumann RB) am Westund Ostrand des Modellgebietes. Die Dichtwände entlang der Oberflächengewässer (Abb. 1) wurden als hydraulisch minder durchlässige Strukturen tiefenabhängig im 
Modell implementiert. Die parallel zu den Dichtwänden verlaufenden Drainagen sowie weitere Drainageleitungen östlich der Salzach und im Bereich Rehhofbrunnen wurden mit interpolierten Festpotenzialen belegt und anhand der Durchflussmengen verifiziert. Die Grundwasserneubildung wurde in Harum et al. (2016) für variable Flurabstände und Vegetationstypen beschrieben und auf Monatsbasis berechnet. Für die vorliegende Studie wurden diese Zeitreihen gemittelt und für unterschiedliche Infiltrationsklassen nach Versiegelungsgrad der Modellelemente bestimmt.

\section{Stochastische Simulation der Materialeigenschaften}

Die Materialeigenschaften des Grundwasserkörpers (z.B. $\mathrm{K}_{\mathrm{f}}$-Werte, Porosität) hängen in erster Linie von der Korngrößenverteilung der fluvialen Sedimentschichten ab. Ausgehend von einer Reihe lithologischer Bohrprofile wurde eine stochastische multiple-point-Simulation (,MPS“) eingesetzt (Strebelle 2002; Hu und Chugunova 2008; Huysmans und Dassargues 2009; dell'Arciprete et al. 2012; Kessler et al. 2013), um eine dreidimensionale Verteilung der zu erwartenden Sedimentassoziationen zu erzeugen. MPS verwendet zur Beschreibung der räumlichen Heterogenität ein konzeptionelles, kategorisiertes 3D-Modell der räumlichen Verteilung der Sedimentklassen und der Lagerungsverhältnisse. Die der Simulation zugrundeliegenden Training Images (TIs) wurden aus Bohrungen, 3D-Variografie und dem digitalen Geländemodell (bspw. alte Mäanderstrukturen) abgeleitet. Daraus wird eine Anzahl konditionaler Realisationen des Simulationsvolumens erzeugt, die alle statistisch korrekt und gleich wahrscheinlich sind. Für die Simulationen standen 43 Senkrechtbohrungen mit Längen von 8,7 bis $107 \mathrm{~m}$ zur Verfügung. Die Oberkante des Simulationsvolumens wird durch die Geländeoberkante de- finiert, und der Stauerhorizont bildet die untere Begrenzung. Die räumliche Auflösung des Simulationsvolumens beträgt $10 \mathrm{~m} \times 10 \mathrm{~m} \times 2 \mathrm{~m}$. Aus allen simulierten Realisationen wurde die wahrscheinlichste 3D-Gesteinsverteilung ermittelt, d.h. jedem Modellelement diejenige Sedimentklasse zugeordnet, welche in 50 Realisationen am häufigsten auftritt. Schließlich wird jedem Element entsprechend der simulierten Sedimentklasse eine assoziierte hydraulische Durchlässigkeit zugewiesen (Jandrisevits et al. 2014; Marschallinger et al. 2015). Diese diskrete Zuweisung stellt letztlich die quantitative Basis für die Parametrisierung des hydraulischen Modells dar. In Abb. 2 ist exemplarisch gezeigt, wie die Sedimentklassen der stochastischen Simulation auf die Modellschichten im Modell übertragen wurden. Dabei wurden jeweils zusammengehörige Schichtpakete zusammengefasst, um der geringeren vertikalen Auflösung im numerischen Modell gerecht zu werden.

Abb. 2a zeigt die Sedimenttypenverteilung der geostatistischen Modellierung für eine Modellschicht. Es sind einige typische sandige Sedimentationsstrukturen wie Sandlinsen ersichtlich, welche in einer kiesig-sandigen Matrix eingebettet sind. Auffällig ist eine im Einflussbereich der Salzach durch die Süd-Nord gerichtete Längung der Sandkörper geprägte Anisotropie. Im Deltabereich der Königseeache wechseln die Sedimente von Westen nach Osten rasch von Kiesen zu Sanden. Die Deltasande der Königseeache entwickeln sich kontinuierlich aus den praktisch reinen Kiesen im übertieften Westbereich und verzahnen mit den Sanden der Salzach. Zudem besteht eine deutliche Schrägschichtung der Deltasedimente der Königseeache. In Abb. 2b sind die Sedimentstrukturen im numerischen Modell in gröberer räumlicher Auflösung nachgebildet, wobei im Staubereich des Kraftwerks die im Liegenden dominanten Feinsande und Schluffe erkenntlich werden.

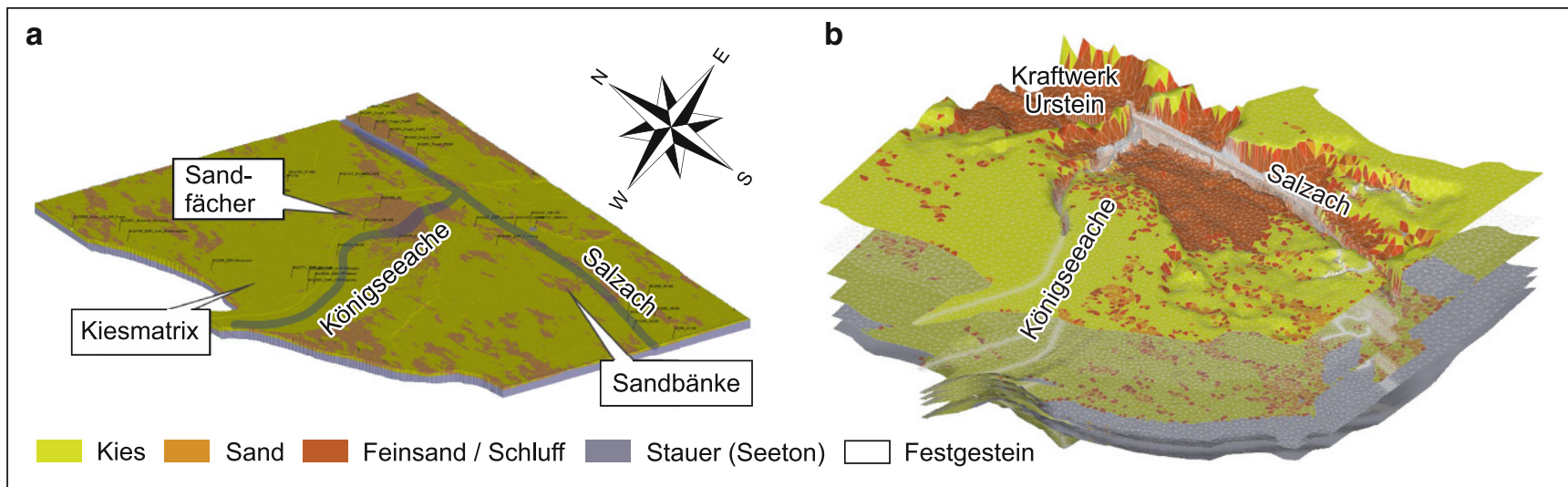

Abb. 2 Implementierung einer stochastischen Sedimentklassenverteilung in das numerische Modell. a zeigt eine exemplarische Simulation mit Hilfe eines multiple-point Algorithmus. In b ist die Integration der simulierten Sedimentklassenverteilung in den Schichtenaufbau des numerischen Modells gezeigt

Fig. 2 Implementation of a sediment class distribution into a numerical model. a illustrates an exemplary simulation using a multiple-point algorithm. In $\mathbf{b}$ the integration of the simulated sediment class distribution into the layered numerical model is shown 


\section{Kalibrierung}

Die stationäre Modellkalibrierung wurde manuell anhand zweier Parameter durchgeführt, der hydraulischen Durchlässigkeit der simulierten Sedimentklassen sowie den Transferraten der Oberflächengewässer. Die kalibrierte Isohypsenverteilung sowie gemittelte Grundwasserstandsmessungen aus dem Jahr 2015 als Zielfunktion sind in Abb. 3 gezeigt. Für die Definition der hydraulischen Durchlässigkeiten ist die räumliche Verteilung der Sedimentklassen gegeben und für alle Modellelemente einer Klasse wurden einheitliche Werte zugewiesen. Diese wurden anhand von Erfahrungswerten aus vorangegangenen Studien (Kersch 2016; Harum et al. 2016; Geoconsult 2017) adaptiert und im Zuge der Kalibrierung angepasst. Die resultierenden $\mathrm{K}_{\mathrm{f}^{-}}$ Werte betragen für Kies $1 \mathrm{E}-03 \mathrm{~m} / \mathrm{s}$, für Sand $1 \mathrm{E}-04 \mathrm{~m} / \mathrm{s}$, für Feinsand/Schluff $2 \mathrm{E}-06 \mathrm{~m} / \mathrm{s}$ und für den Grundwasser Stauer (Salzburger Seeton) 5E-07 m/s. Die Unsicherheiten werden mit 0,5 bis 1 Größenordnung beziffert, da eine größere Variation der hydraulischen Durchlässigkeiten eine gravierende Verformung des Strömungsbildes und damit eine Verschlechterung des Kalibrierungsergebnisses nach sich zieht. Das Verhältnis vertikaler zu horizontaler Durchlässigkeit wurde mit 1:10 angesetzt.
Der Wasseraustausch zwischen Oberflächengewässer und Grundwasser wird mittels Transferrate definiert, welche sich aus dem Quotienten aus hydraulischer Durchlässigkeit der Sohlschicht sowie deren Mächtigkeit ergibt. Aus der Kalibrierung ergeben sich für unterschiedliche Fließabschnitte Werte zwischen 1E-04 1/s und 1E-06 1/s. Im Bereich des Sandfächers der Königseeache (Abb. 2) steigt demnach der hydraulische Gradient an, und die Infiltrationsrate der Königseeache resultiert in diesem Bereich zwei Größenordnungen geringer als oberstrom des Sandfächers. Die zunehmend sandigen Sedimente im Mündungsbereich der Königseeache wirken sich daher auf das Fließgeschehen in Richtung der Mündung aus. Die Kalibrierung mittels Transferrate ermöglichte die steilen Gradienten im Bereich des Kraftwerks Urstein sowie die Infiltration der Königseeache in Niederalm.

In Anbetracht des durch die Baumaßnahmen komplexen Strömungsfeldes liefert das Modell ein zufriedenstellendes Abbild des Grundwasserniveaus. Im Umfeld der Rehhofsiedlung im Zentrum des Modellgebiets stimmen die berechneten Grundwasserhöhen gut mit den gemessenen Werten überein. Die berechneten Abweichungen betragen etwa $20-30 \mathrm{~cm}$. Etwas größere Abweichungen treten im Nahbereich der Oberflächengewässer Salzach und Königseeache auf, da hier steile Gradienten durch die hydrauli-

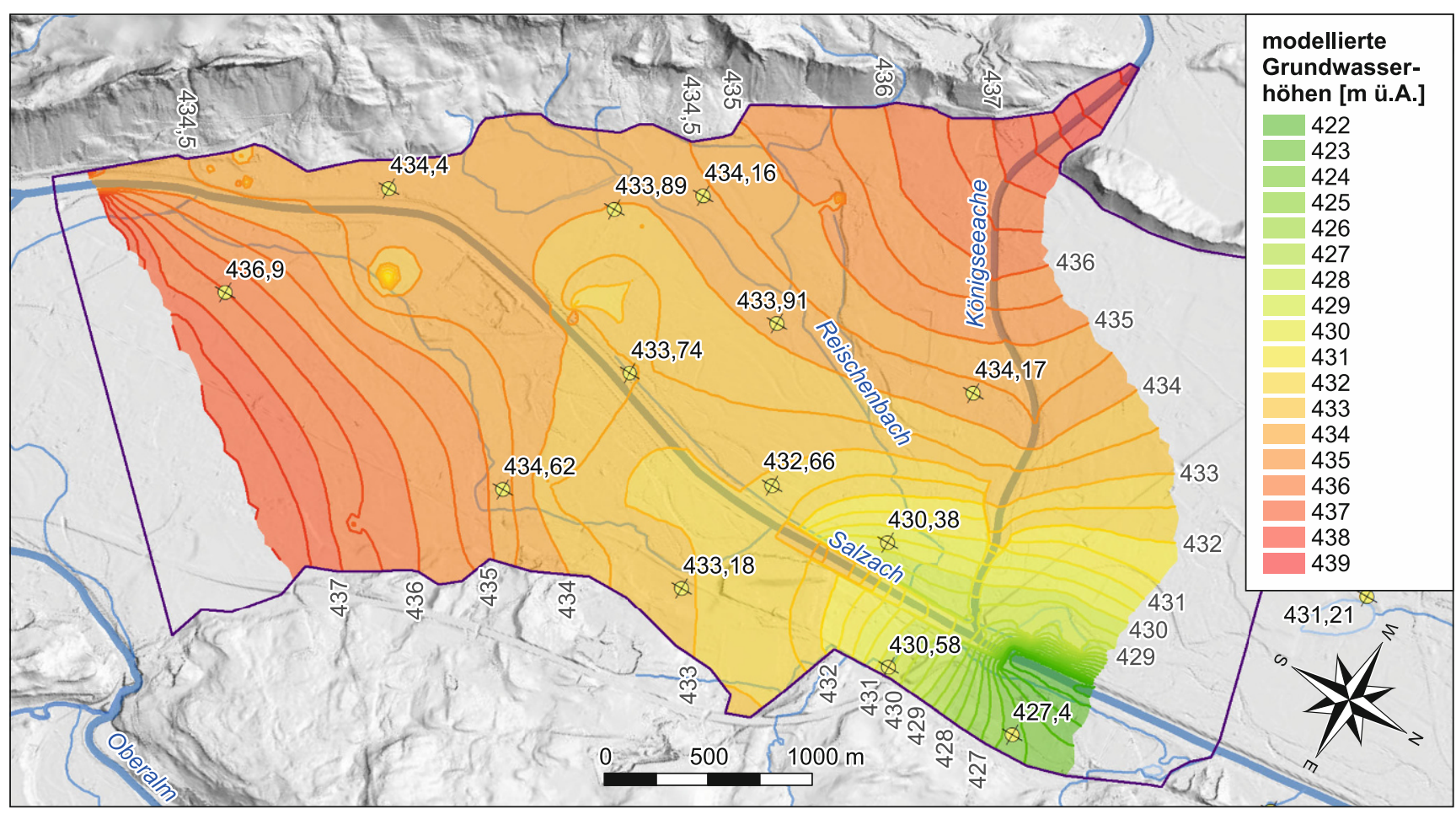

Abb. 3 Grundwassergleichenplan des kalibrierten Referenzmodells. Für die Kalibrierung wurde eine Stichtagsmessung aus dem Jahr 2015 herangezogen (gelb markierte Messstellen). Die nicht eingefärbten Bereiche des Modellgebietes sind inaktiv und wurden nicht in die Berechnung einbezogen

Fig. 3 Groundwater contour plan of the calibrated reference model. For model calibration a reference date measurement of the year 2015 was used. The transparent zones inside the modelling area are set inactive and are thus not subject to the computation 
schen Baumaßnahmen auftreten. Eine Feinkalibrierung erschien hier wenig sinnvoll, da die Fließkontrolle durch die internen Randbedingungen potenzielle Wasserstandsänderungen dämpfen oder ganz verhindern würde. Insgesamt ist die Größenordnung der berechneten Grundwasserstandsschwankungen jedoch als realistisch einzuordnen. Die Kalibrierung anhand mehrerer Parameter birgt das Risiko der Mehrdeutigkeit der Ergebnisse. In diesem Fall ist vor allem der Wertebereich der hydraulischen Durchlässigkeiten durch die vorangegangenen Studien und durch eine homogene Korngrößensortierung beschränkt. Weit größere Unsicherheiten sind bei den Transferraten der Oberflächengewässer zu erwarten, und haben sich auch als die sensitiveren Kalibrierungsparameter erwiesen.

\section{Modellszenarien}

Ausgehend vom kalibrierten Referenzmodell wurden mehrere Modellszenarien konzipiert und berechnet. Die Szenarien analysieren einerseits die Sensitivität des Grundwasserkörpers im Hinblick auf Variationen der baulichen, klimatischen und hydraulischen Gegebenheiten und repräsentieren gleichzeitig mögliche zukünftige Entwicklungen im Untersuchungsgebiet.

\section{Zunahme Siedlungsdichte (a)}

In der näheren Zukunft wird es möglicherweise $\mathrm{zu}$ einer weitreichenden Umwidmung und folglich auch zur Bebauung von Grünland zwischen Rif und Rehhof kommen (siehe Abb. 4a). Es ist daneben mit einer zunehmenden Verdichtung der Siedlungsflächen in den Gemeinden Rif und Niederalm zu rechnen. Sind im jetzigen Zustand knapp $40 \%$ der Flächen versiegelt, wurde im Berechnungsszenario a durch Bebauung von Brachflächen und Verdichtungen ein Versiegelungsgrad von $70 \%$ zwischen Niederalm und Rehhof zugrunde gelegt. Ein hoher Versiegelungsgrad wirkt sich unmittelbar auf den meteorischen Grundwassereintrag aus. Auf Dachflächen und Parkplätzen beispielsweise ist die Evapotranspiration im Vergleich zu Grünflächen deutlich reduziert, und das abgeführte Niederschlagswasser muss im Normalfall örtlich versickert werden und wird so dem Grundwasserleiter zugeführt. Im Modell wird dieser Umstand durch eine erhöhte Grundwasserneubildung abgebildet (Beaufschlagung 40\%, Grünland $830 \mathrm{~mm} / \mathrm{a}$, versiegelte Flächen $1180 \mathrm{~mm} / \mathrm{a})$.

\section{Strömungshindernis Tiefbau (b)}

Im Szenario b wurde die Flächenversiegelung um den Einfluss des Grundwasseranstaus und das Fehlen von strömungswirksamen Porenvolumen durch Tiefgeschosse er- weitert. Es wurde zugrunde gelegt, dass die gesamte bebaute Fläche mit hydraulisch undurchlässigen Tiefgeschossen ausgestattet ist. Numerisch wurde eine zuoberst liegende Modellschicht von 2,5 m Mächtigkeit mit entsprechender hydraulisch undurchlässiger Charakteristik ergänzt. Um dem Umstand Rechnung zu tragen, dass nur ein Teil der besiedelten Fläche unterkellert ist, wurde lediglich ein Drittel der Elemente in den entsprechenden Gebieten als hydraulisch undurchlässig markiert. Diese wurden nach dem Anteil der Dachflächen je Modellelement ausgewählt. Die Versickerung von Niederschlagswasser auf diesen Elementflächen wurde mit Hilfe von Infiltrationsbrunnen in tieferen Schichten berücksichtigt.

\section{Dysfunktionale Drainagen (c)}

Laut Grundwassermessungen wurde der größte Grundwasseranstieg entlang der Salzach in den Messstellen Taxach Br13, Taxach B127 und AU B134 (Abb. 1) beobachtet. Diese haben möglicherweise dieselbe Ursache des Anstiegs. Um den Einfluss der parallel zur Salzach verlaufenden Drainage zu untersuchen, wurde im Szenario c eine minder funktionale Drainage berücksichtigt, hervorgerufen beispielsweise durch eine Verockerung des Aquifers im unmittelbaren Nahbereich der Uferdrainage. Um einen solchen Zustand im Modell abbilden zu können, wurden die hydraulischen Durchlässigkeiten in einem schmalen Streifen im Zustrom Bereich der Drainage um den Faktor 10E-02 heruntergesetzt.

\section{Flussrandbedingung Drainage (d)}

Die Drainagen entlang der Oberflächengewässer wurden im Referenzmodell mittels definierter Festpotenziale implementiert. Diese Randbedingung eliminiert jedoch etwaige Grundwasserschwankungen im Nahbereich der Salzach weitestgehend, daher wurden die Drainagen alternativ mit einer Flussrandbedingung belegt. Die Potenzialhöhe der Drainage wurde gemäß manuell beobachteter Abflussmenge in eine Flussrandbedingung übersetzt. Bei einer Begehung vor Ort wurde der Drainageabfluss mit 3501/s abgeschätzt. Der berechnete Wert aus dem Referenzmodell beträgt rund 3001/s und liegt im Rahmen der Messgenauigkeit.

\section{Zunahme Niederschlag (e)}

Aufzeichnungen der Tagesniederschlagssummen an den beiden Messstellen „Salzburg Freisaal“ und „Salzburg Flughafen“ (ZAMG 2019) lassen für den Zeitraum von 1987 bis 2017 keine signifikanten Änderungen der Jahresniederschlagsmengen erkennen. In Fliß et al. (2021) werden ähnliche Auswertungen für den Raum Süddeutsch- 
land beschrieben, als im Zeitraum 1951 bis 2019 kein langjähriger Trend, jedoch eine große Variabilität der Jahresniederschlagssummen beobachtet wurden. Für das Niederschlagsszenario wurden statt gemessener Daten simulierte Änderungen der Niederschlagssummen nach den „repräsentativen Konzentrationspfaden“ (RCP, Moss et al. 2010; Meinshausen et al. 2011; Stocker et al. 2013) für das Land Salzburg herangezogen. Dies repräsentiert kein umfassend parametrisiertes Klimaszenario, ermöglicht aber eine semi-quantitative Analyse eines tendenziellen Niederschlagszuwachses auf den Grundwasserkörper. Dabei wurden zwei Modellrechnungen der Niederschlagsentwicklung im Land Salzburg berücksichtigt (Chimani et al. 2016). Die erste geht von erfolgreichen Klimaschutzmaßnahmen aus (RCP 4,5) und die zweite von einer ,weiter-wie-bisher"Entwicklung (RCP 8,5; Riahi et al. 2011). Die prognostizierte maximale Zunahme der Niederschlagssumme (worstcase) beträgt für den Zeitraum 2021-2050+7,9\% für RCP 4,5 und $+11,9 \%$ für RCP 8,5. Für den Zeitraum 2071-2100 beträgt die prognostizierte Zunahme $+12,1 \%$ für RCP 4,5 und $+18,1 \%$ für RCP 8,5 (Chimani et al. 2016). Der erhöhte meteorologische Eintrag in den Grundwasserkörper wurde mit einer flächengemittelten Beaufschlagung der Grundwasserneubildung im Modell integriert. Dies entspricht im Siedlungsgebiet exakt dem Betrag der Niederschlagsveränderung, d.h. keine zusätzliche Verdunstung tritt auf. Auf dem Grünland und im Wald ist ein Anteil von etwa $30 \%$ zusätzlicher Verdunstung bezogen auf die Niederschlagsveränderung berücksichtigt.

\section{Abflussschwankung Königseeache (f)}

Der Wasserstand der Königseeache zeigt einen Jahresgang aufgrund eines variablen Abflusses des Königsees und schwankenden Zuflüssen. In Harum et al. (2016) wurden anhand von Schlüsselkurven Wasserstands-Abflussbeziehungen erstellt. Da die Schwankungen jedoch keinen langjährigen Trend zeigen, wurde für die Sensitivitätsanalyse der Wasserstand nicht auf Basis der Abflussdaten variiert, sondern ein künstlicher, gemittelter Anstieg des Wasserstands zugrunde gelegt. Im Modell wurden höhere Wasserstände von $-10 \mathrm{~cm},+10 \mathrm{~cm}$ und $+20 \mathrm{~cm}$ im Vergleich zum gemittelten Wasserstand für den Flussabschnitt oberstrom des Rückstaubereiches des Kraftwerks Urstein zugrunde gelegt.

\section{Transferrate Königseeache (g)}

Ein vermeintlich großer Unsicherheitsfaktor des Modells ist die Infiltrationsrate aus den Oberflächengewässern, insbesondere der Königseeache. Im Berechnungsszenario g wurde die Infiltrationsrate von Uferregionen, in denen sedimentäre Strukturen wie Sandfächer oder Schrägschichten auf- treten, über die Berandung des Flussbettes räumlich variiert. Für jeden dieser Bereiche wurden variable hydraulische Durchlässigkeiten der Kolmatierungsschicht angenommen (siehe Parameter phi in Abb. 4g, h).

\section{Ergebnisse und Diskussion}

Die Szenarien $(a-g)$ wurden anhand der Variation eines oder mehrerer Modellparameter auf ihre hydraulischen Auswirkungen getestet. Die Ergebnisse sind in Abb. 4 anhand von Grundwasserdifferenzen im Vergleich zum kalibrierten Referenzmodell dargestellt, wobei sich die Interpretation auf das Modellkerngebiet konzentriert. Bei einer Zunahme der Siedlungsdichte, d.h. zunehmender Flächenversiegelung (Szenario $a$, Abb. 4a), kommt es zu einer Steigerung der Sickerwassermenge und einer positiven Bilanz des quantitativen Grundwasserzustandes. Das Niederschlagswasser von Dachflächen wird gesammelt, in den Untergrund abgeleitet und damit der Evapotranspiration entzogen. Durch die punktuelle Beaufschlagung der Grundwasserneubildung kommt es zu einem Grundwasseranstieg von $0-5 \mathrm{~cm}$, wobei die größten Effekte am Westrand und im Zentrum des Modellkerngebietes auftreten. In diesem Bereich sind die Flurabstände mit $0,5-2,0 \mathrm{~m}$ am geringsten, und der stabilisierende Effekt der Festpotenziale der umliegenden Drainagen fehlt. Dass der berechnete Anstieg eher gering ausfällt, liegt an einem Anteil von lediglich etwa $20 \%$ der Grundwasserneubildung an der Gesamtgrundwasserbilanz. Diese beträgt rund 17001/s für die Durchströmung des Modellgebietes. Davon entfallen 3701/s auf den Salzachtalaquifer, 3301/s auf die Grundwasserneubildung und 6401/s auf die Infiltration der beiden Fließgewässer. Der Rest verteilt sich auf die Drainagen entlang der beiden Fließgewässer.

Sofern die Bebauung mit Tiefgeschossen ausgestattet ist, wird die Grundwasserströmung in Regionen mit hohem Grundwasserstand durch die Tiefbauten behindert. Die Berechnungen (Szenario b, Abb. 4b) zeigen, dass es lokal zu Grundwasserschwankungen von $5-10 \mathrm{~cm}$, stellenweise sogar zu einem Anstieg von $+20 \mathrm{~cm}$ kommen kann. Eine Summenwirkung des Grundwasseranstaus ist insbesondere dann zu erwarten, sofern eine dichte Bebauungsstruktur mit einer vollflächigen Unterkellerung (Wohnkomplexe mit Tiefgaragen) besteht. Im Modell wird dieser Effekt etwas überschätzt, da eine exakte Diskretisierung der Gebäudeumrisse aufgrund der groben Diskretisierungsweite nicht möglich war.

Der dokumentierte Grundwasseranstieg von $20-30 \mathrm{~cm}$ in den salzachnahen Grundwassermessstellen (Au B134, Taxach Br13, Taxach B127) kann am besten mit reduzierten $\mathrm{K}_{\mathrm{f}}$-Werten im Zustrombereich der Drainage nachgerechnet werden (Szenario c, Abb. 4c). Daraus schließt sich, dass ei- 


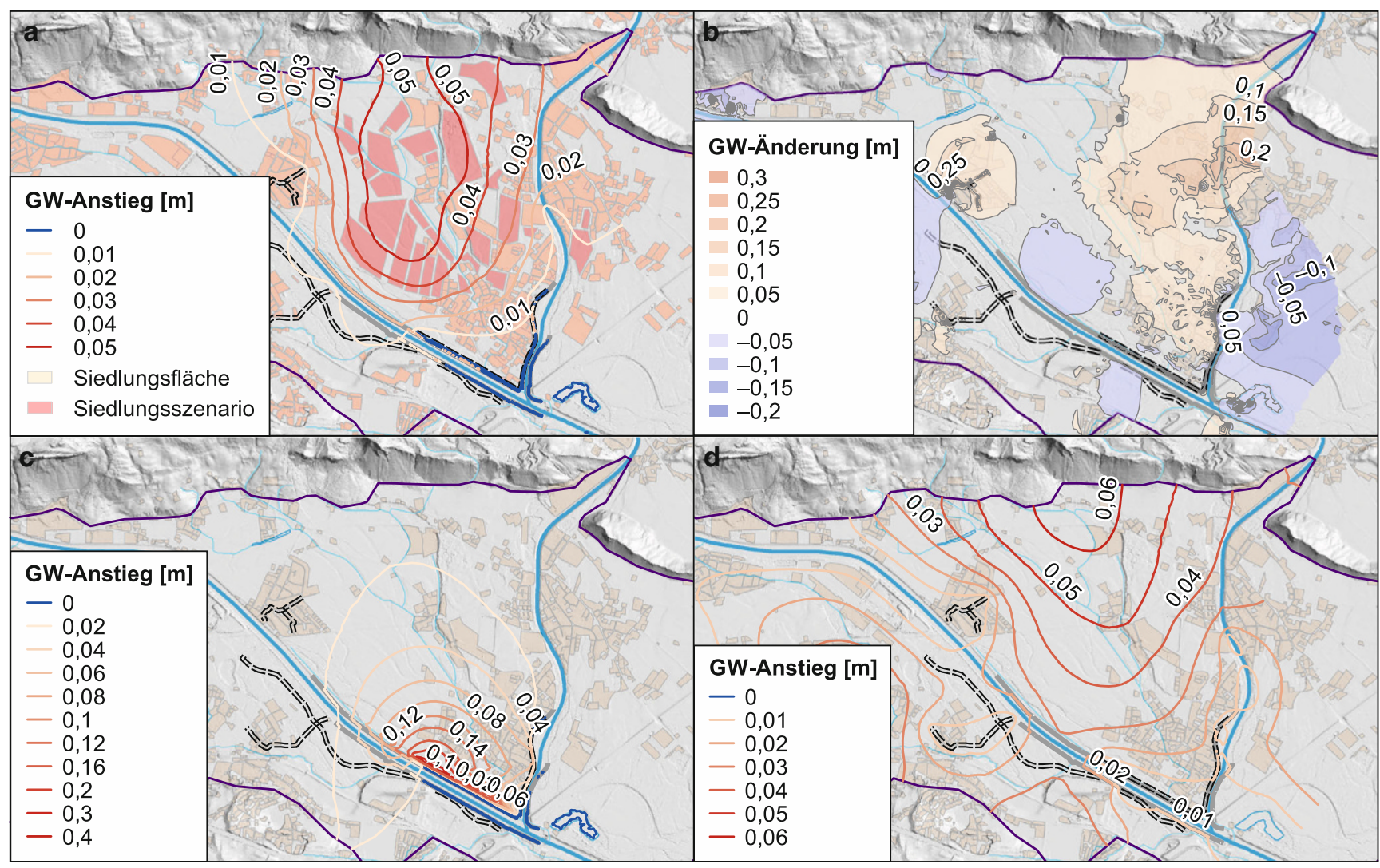

Abb. 4 Darstellung der Grundwasserdifferenzen der Modellszenarien a-g. a Szenario a - Zunahme Siedlungsdichte; b Szenario b - Strömungshindernis Tiefbau; c Szenario c und d - dysfunktionale Drainagen mit unterschiedlichen Randbedingungen; d und e Szenario e - Zunahme Niederschlag im Zeitraum bis 2050 (d) und bis 2100 (e); f Szenario f - Abflussschwankungen Königseeache; g und h Szenario g - Interaktion Königseeache mit unterschiedlichen Transferraten phi

Fig. 4 Illustration of the groundwater differences of the model scenarios a-g. a scenario a - increase of settlement density; $\mathbf{b}$ scenario b - underground flow barriers; $\mathbf{c}$ scenario $\mathrm{c}$ and $\mathrm{d}-$ dysfunctional drainages with varying boundary conditions; $\mathbf{d}$ and $\mathbf{e}$ scenario e - precipitation increase for the period until 2050 (d) and until 2100 (e); f scenario f - discharge fluctuations of the Königseeache; $\mathbf{g}$ and $\mathbf{h}$ scenario $\mathrm{g}-$ interaction with Königseeache with varying transfer rates phi

ne minder funktionale Drainage den Rückstau des Grundwassers mindestens begünstigt. Dieser Effekt könnte beispielsweise durch eine Verockerung der Drainageöffnungen oder durch Erreichen der Kapazitätsgrenze der Drainage hervorgerufen werden. Im vorliegenden Fall, in dem eine freie Grundwasserströmung durch hydraulische Maßnahmen stark eingeschränkt ist, verlieren nicht funktionale Bauwerke ihre Pufferwirkung und führen zu mittelfristigen Änderungen des Grundwasserniveaus. Daher lohnt es sich im Rahmen der Sensitivitätsanalyse auch die hydraulischen Randbedingungen der Bauwerke zu testen, ob die zugrunde liegenden Annahmen zutreffend sind. Am Beispiel der Drainage wurde im Szenario $d$ die Randbedingung 1. Ordnung in eine Flussrandbedingung 2. Ordnung übersetzt. Das Modell zeigt bei Wechsel auf eine Flussrandbedingung eine ähnliche Potenzialverteilung wie im Referenzmodell.

Höhere Niederschlagsmengen, hervorgerufen durch ein verändertes Klima, haben im Untersuchungsgebiet ebenfalls einen geringen Einfluss auf den Grundwasserhaushalt (Szenario e, Abb. 4d und e). In Abb. 4d für den Zeitraum
2021-2050 und Abb. 4e für den Zeitraum 2071-2100 sind nur die berechneten Grundwasseranstiege für den ungünstigsten Fall des RCP 8,5 gezeigt. Für den RCP 8,5 betragen die maximal möglichen Grundwasseranstiege etwa $5 \mathrm{~cm}$ bis 2050 und $18 \mathrm{~cm}$ bis zum Jahr 2100. Der Anstieg tritt jedoch großflächig auf und liegt im Mittel bei rund 3 und $10 \mathrm{~cm}$ respektive. Die Änderungen für die erwarteten Niederschlagswerte des RCP 4,5 betragen nur wenige Zentimeter. Ähnlich dem Szenario a) wirkt der geringe Beitrag der Neubildung zur Gesamtgrundwasserbilanz stabilisierend auf den Grundwasserkörper. Eine geringfügig höhere Verdunstung für Grünland und Wald wurde für diese Berechnung zugrunde gelegt. Bei Berücksichtigung weiterer klimatischer Parameter wie beispielsweise stärkerer Sonneneinstrahlung könnte der Grundwasseranstieg sogar noch geringer ausfallen. Ebenfalls analog zur Beaufschlagung in Szenario a), treten die höchsten Anstiege im Bereich der westlichen Modellrandbegrenzung auf, da hier die Senkenfunktion der Drainage am wenigsten wirksam ist. 


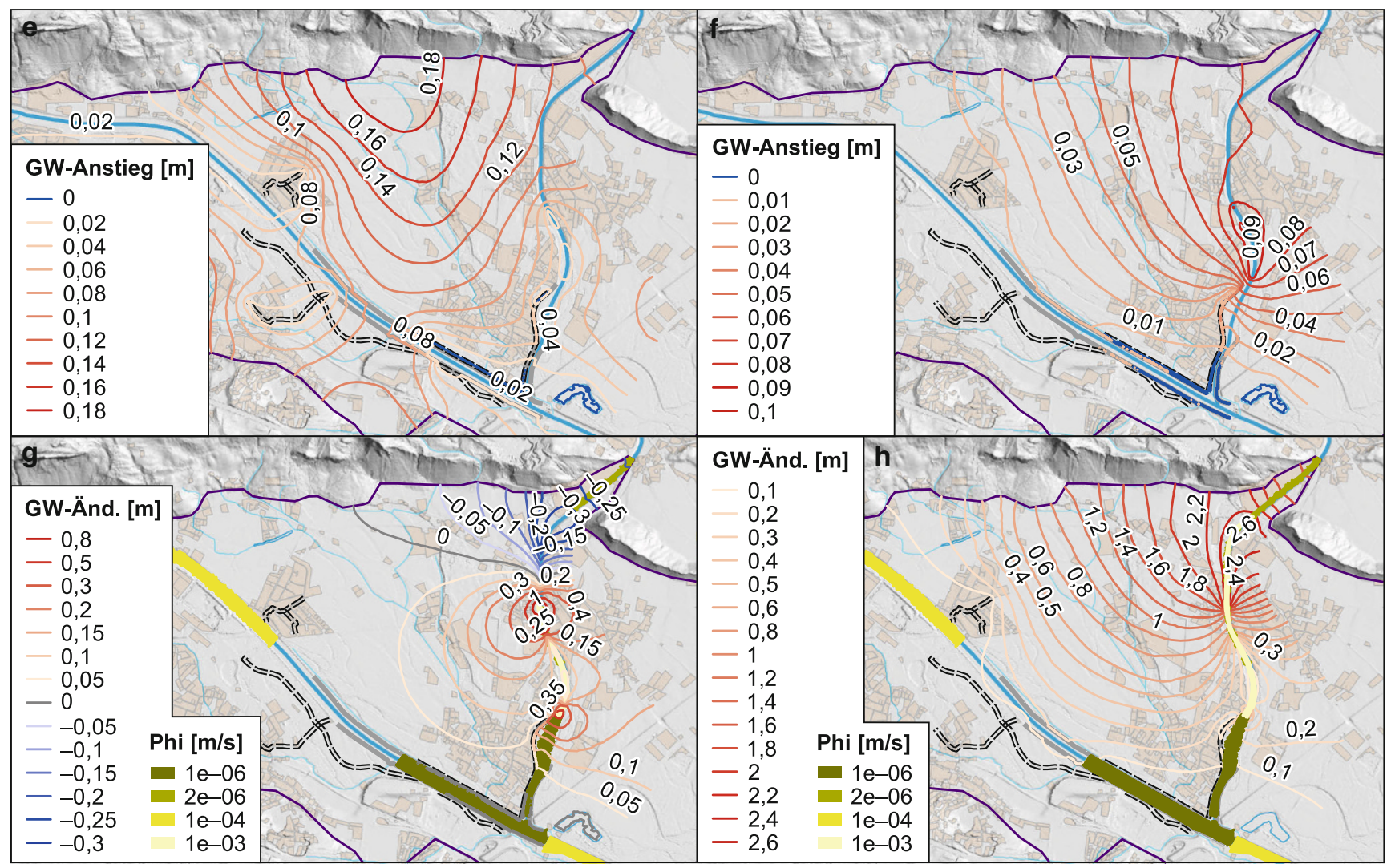

Abb. 4 (Fortsetzung)

Fig. 4 (continued)

Die Königseeache als interaktives Oberflächengewässer erweist sich als sensitiver Faktor für den Aquifer. Ein Flusswasserspiegel von $+10 \mathrm{~cm}$ wirkt sich im Nahbereich des Fließgewässers mit $6-7 \mathrm{~cm}$ Grundwasseranstieg aus, wobei geringfügige Auswirkungen von wenigen Zentimetern im gesamten Siedlungsgebiet Niederalm berechnet wurden (Szenario $f$, Abb. 4f). Bei einem Anstieg von $+20 \mathrm{~cm}$ sind die Auswirkungen lokal entsprechend höher, und der Grundwasserstand steigt in der Siedlung Niederalm und auch in Teilen von Rif um 8-10 cm an. Die Anstiege zeigen eine gute Konnektivität der Wasserkörper und verdeutlichen die Anfälligkeit des Aquifers beispielsweise im Hochwasserfall. Im Szenario $g$ (Abb. $4 \mathrm{~g}$ und $4 \mathrm{~h}$ ) werden letztlich die Transferraten räumlich variiert, um die Zonen starker Interaktion einzugrenzen. In $\mathrm{Abb} .4 \mathrm{~g}$ ist ersichtlich, dass es bei einer hohen Transferrate phi (hellgelber Bereich) unmittelbar oberstrom des Rückstaubereiches des Kraftwerks Urstein vor allem im Uferbereich zu starken Grundwasserschwankungen kommt. Möglicherweise puffert die flussparallele Drainage weitreichendere Auswirkungen. Ein großflächiger Grundwasseranstieg von mehreren Dezimetern und stellenweise bis zu $2 \mathrm{~m}$ tritt auf, wenn die gut durchlässige Transferstrecke oberstrom der Königseeache erweitert wird (Abb. 4h). Hier existiert im Bereich des Zu- flusses der Königseeache ins Modellgebiet ein kritischer Fließabschnitt. Falls in diesem Abschnitt die Transferrate ansteigt (bspw. aufgrund von Spülstößen, Sohlbrüchen oder Überschwemmung nicht kolmatierter Uferbereiche) kommt es zu einer unterirdischen Flutung des Modellkerngebietes. Dieser Teil der Fließstrecke muss daher im Hinblick auf eine Stabilisierung des Grundwasserniveaus besondere Beachtung finden. Eine räumlich differenzierte Betrachtung der Fließgewässerinteraktion ist daher elementar für das Verständnis der Dynamik des Grundwasserkörpers. Es hat sich dabei als zielführend erwiesen die Sensitivitätsanalyse für imaginäre Wasserstände zu berechnen anstatt eine zeitabhängige Korrelation von Grund- und Oberflächengewässer zugrunde zu legen. Die teils geringen Schwankungen der Königseeache, die unterschiedlichen Messintervalle sowie die Pufferwirkung des Aquifers gleichen die simulierten Wechselwirkungen des Grund- und Oberflächengewässers aus und ließen aus einer instationären Betrachtungen wenige belastbare Erkenntnisse zu. 


\section{Schlussfolgerungen}

Die Modellierungen haben gezeigt, dass ein langfristiger Grundwasseranstieg das Ergebnis multipler Faktoren bzw. eine Kombination aus diesen sein kann. Auffällig ist, dass eine rasche Flächenversiegelung wachsender Siedlungen inklusive einer Zunahme der Infiltrationsmengen durch lokale Sickerflächen einen eher geringen Einfluss auf den Grundwasserhaushalt hat. Daran ändert auch eine weiträumige Unterkellerung von Gebäuden nichts, solange eine Summenwirkung des Grundwasseranstaus durch große, eng stehende Gebäudekomplexe verhindert wird. Ähnliches gilt für mengenbedingte Variationen im meteorologischen Eintrag. Diese Tendenz lässt sich mit der Massenbilanz belegen, in der die Grundwasserneubildung im Vergleich zum flächengemittelten Durchfluss und Infiltration von Oberflächenwasser einen vergleichsweise kleinen Beitrag leistet. Diese Tatsache ist spezifisch für das Untersuchungsgebiet Rif. Bei einer Übertragung der vorgestellten Ergebnisse auf andere Gebiete müssen stets die naturräumlichen und hydraulischen Gegebenheiten auf Vergleichbarkeit geprüft werden. Wesentlich für das quantitative Grundwassermanagement sind hingegen die hydraulischen Randbedingungen der mit dem Aquifer in Kontakt stehenden Oberflächengewässer, inklusive der damit verbundenen Interaktion. $\mathrm{Zu}$ diesem Schluss kommen beispielsweise auch Hanauer und Möbius (2020), die den Grundwasserstrom in einem alpinen Talkessel im benachbarten Ruhpolding modelliert haben. Im hiesigen Fall weist die Königseeache mit ihrer variablen Wasserführung unterschiedliche Kolmatierungscharakteristiken im Bett und an der Berandung auf, welche je nach Wasserstand mehr oder weniger Wasseraustausch erlauben. Der Abfluss der Königseeache inklusive möglicher Hochwässer und der damit verbundenen Dynamik der Sohlsedimente ist daher in Rif einer der sensitivsten Parameter für das Grundwassermanagement der Gemeinde.

Der Ansatz einer numerischen Analyse sensitiver Parameter der Grundwasserdynamik kann eine nützliche Methode für eine daran anknüpfende ingenieurtechnische Planung und Bewertung im Rahmen genehmigungsrechtlicher Verfahren sein. Basierend auf dem Wissen spezifischer grundwasserhydraulischer Gegebenheiten kann ein vorausschauendes Grundwassermanagement realisiert werden.

Danksagung Wir danken dem Land Salzburg für die Unterstützung der Forschungsarbeiten und den wissenschaftlichen Gutachtern für die konstruktiven Reviews bei der Erstellung dieses Beitrags.

Funding Open Access funding enabled and organized by Projekt DEAL.

Open Access Dieser Artikel wird unter der Creative Commons Namensnennung 4.0 International Lizenz veröffentlicht, welche die Nutzung, Vervielfältigung, Bearbeitung, Verbreitung und Wiedergabe in jeglichem Medium und Format erlaubt, sofern Sie den/die ursprüng- lichen Autor(en) und die Quelle ordnungsgemäß nennen, einen Link zur Creative Commons Lizenz beifügen und angeben, ob Änderungen vorgenommen wurden.

Die in diesem Artikel enthaltenen Bilder und sonstiges Drittmaterial unterliegen ebenfalls der genannten Creative Commons Lizenz, sofern sich aus der Abbildungslegende nichts anderes ergibt. Sofern das betreffende Material nicht unter der genannten Creative Commons Lizenz steht und die betreffende Handlung nicht nach gesetzlichen Vorschriften erlaubt ist, ist für die oben aufgeführten Weiterverwendungen des Materials die Einwilligung des jeweiligen Rechteinhabers einzuholen.

Weitere Details zur Lizenz entnehmen Sie bitte der Lizenzinformation auf http://creativecommons.org/licenses/by/4.0/deed.de.

\section{Literatur}

Arnoux, M., Halloran, L.J.S., Berdat, E., Hunkeler, D.: Characterizing seasonal groundwater storage in alpine catchments using timelapse gravimetry, water stable isotopes and water balance methods. Hydrol. Process. 34(22), 4319-4333 (2020). https://doi.org/ 10.1002/hyp. 13884

Arnoux, M., Brunner, P., Schaefli, B., Mott, R., Cochand, F., Hunkeler, D.: Low-flow behavior of alpine catchments with varying quaternary cover under current and future climatic conditions. J. Hydrol. Reg. Stud. 592, 125591 (2021). https://doi.org/10.1016/j.jhydrol. 2020.125591

Barua, S., Cartwright, I., Dresel, P.E., Daly, E.: Using multiple methods to investigate the effects of land-use changes on groundwater recharge in a semi-arid area. Hydrol. Earth Syst. Sci. 25(1), 89-104 (2021). https://doi.org/10.5194/hess-25-89-2021

Bender, S., Groth, M., Viktor, E.: Auswirkungen des Klimawandels auf die zukünftige Grundwassernutzung - Betroffenheiten, Handlungsbedarfe und Lösungsansätze. Grundwasser - Zeitschrift der Fachsektion Hydrogeologie 26(1), 61-72 (2021). https://doi.org/ 10.1007/s00767-020-00465-9

Bloomfield, J.P., Marchant, B.P.: Analysis of groundwater drought building on the standardised precipitation index approach. Hydrol. Earth Syst. Sci. 17(12), 4769-4787 (2013). https://doi.org/ 10.5194/hess-17-4769-2013

Blöschl, G., Schöner, W., Kroll, H., Blaschke, A.P., Böhm, R., Haslinger, K., Kreuzinger, N., Merz, R., Parajka, J., Salinas, J., Viglione, A.: Anpassungsstrategien An Den Klimawandel für Österreichs Wasserwirtschaft: Ziele und Schlussfolgerungen der Studie für Bund und Länder. Österr Wasser- Abfallw I-2, 1-10 (2011)

Blöschl, G., Blaschke, A.P., Haslinger, K., Hofstätter, M., Parajka, J., Salinas, J., Schöner, W.: Auswirkungen der Klimaänderung auf Österreichs Wasserwirtschaft - ein aktualisierter Statusbericht. Österr Wasser- und Abfallw 70(9-10), 462-473 (2018). https:// doi.org/10.1007/s00506-018-0498-0

Blumstock, M., Tetzlaff, D., Dick, J.J., Nuetzmann, G., Soulsby, C.: Spatial organization of groundwater dynamics and streamflow response from different hydropedological units in a montane catchment. Hydrol. Process. 30(21), 3735-3753 (2016). https:// doi.org/10.1002/hyp. 10848

Bonat, M., Lucianetti, G., Mastrorillo, L.: The role of alpine valley fill deposits for groundwater storage (Dolomites, Italy). Grundwasser 25(1), 3-14 (2020). https://doi.org/10.1007/s00767-019-00438-7

Brandecker, H.: Hydrogeologie des Salzburger Beckens. Steir. Beitr. Hydrogeol. 26, 5-39 (1974)

Braunstingl, R., Pestal, G.: Geologische Karte von Salzburg. Geologie der österreichischen Bundesländer. Geologische Bundesanstalt, Wien (2005)

Chen, Z., Hartmann, A., Wagener, T., Goldscheider, N.: Dynamics of water fluxes and storages in an Alpine karst catchment under current and potential future climate conditions. Hydrol. Earth 
Syst. Sci. 22(7), 3807-3823 (2018). https://doi.org/10.5194/hess22-3807-2018

Chimani, B., Heinrich, G., Hofstätter, M., Kerschbaumer, M., Kienberger, S., Leuprecht, A., Lexer, A., Peßenteiner, S., Poetsch, M., Salzmann, M., Spiekermann, R., Switanek, M., Truhetz, H.: ÖKS15 Klimafactsheet: Klimaszenarien für das Bundesland Salzburg bis 2100. ÖKS, 5. Land Salzburg, Austria (2016)

Czernin-Chudenitz, C., Hitsch, E., Kirchner, E.C., Prodinger, F., Schmidt, G., Üblagger, G.: Untersuchungen zu Fragen der Wasserentsorgung im Gebiet Leopoldskron Moos Gneis. In: Umweltschutzprogramm, Bd. I-XV, S. 1-322. Magistrat Salzburg, Salzburg (1978)

dell'Arciprete, D., Bersezio, R., Felletti, F., Giudici, M., Comunian, A., Renard, P.: Comparison of three geostatistical methods for hydrofacies simulation: A test on alluvial sediments. Hydrogeol J 20(2), 299-311 (2012). https://doi.org/10.1007/s10040-011-0808-0

Diersch, H.-J.: Feflow-Finte Element Modeling of Flow, Mass and Heat Transport in Porous and Fractured Media, 1. Aufl. Springer, Berlin, Heidelberg, New York, S. 996 (2014) https://doi.org/10. 1007/978-3-642-38739-5

Donadel, A.K., Höfer-Öllinger, G., Frimmel, H.E., Schrott, L.: Evolution of late- to postglacial river confluences in overdeepened alpine valleys-Saalach and Königsseeache (Austria). Austrian J Earth Sci 107, 60-73 (2014)

Eckhardt, K., Ulbrich, U.: Potential impacts of climate change on groundwater recharge and streamflow in a central European low mountain range. J. Hydrol. Reg. Stud. 284(1), 244-252 (2003). https://doi.org/10.1016/j.jhydrol.2003.08.005

Epting, J., Michel, A., Affolter, A., Huggenberger, P.: Climate change effects on groundwater recharge and temperatures in Swiss alluvial aquifers. J. Hydrol. Reg. Stud. 11, 100071 (2021). https://doi. org/10.1016/j.hydroa.2020.100071

Fette, M., Hoehn, E., Wehrli, B.: Infiltration von Flusswasser ins Grundwasser. Wasser Energ. Luft 11(12), 301-304 (2004)

Finger, D., Hugentobler, A., Huss, M., Voinesco, A., Wernli, H., Fischer, D., Weber, E., Jeannin, P.-Y., Kauzlaric, M., Wirz, A., Vennemann, T., Hüsler, F., Schädler, B., Weingartner, R.: Identification of glacial meltwater runoff in a karstic environment and its implication for present and future water availability. Hydrol. Earth Syst. Sci. 17(8), 3261-3277 (2013). https://doi.org/10.5194/hess17-3261-2013

Fliß, R., Baumeister, C., Gudera, T.: Auswirkungen des Klimawandels auf das Grundwasser und die Wasserversorgung in Süddeutschland. Grundwasser 26(1), 33-45 (2021). https://doi.org/10.1007/ s00767-021-00477-z

Geoconsult: Grundwassermodellierung BVH Webereiweg 2, HalleinRif, Austria (2017)

Gobiet, A., Kotlarski, S., Beniston, M., Heinrich, G., Rajczak, J., Stoffel, M.: $21^{\text {st }}$ century climate change in the European Alps-A review. Sci. Total Environ. 493, 1138-1151 (2014). https://doi.org/ 10.1016/j.scitotenv.2013.07.050

Haas, J.C., Birk, S.: Trends in Austrian groundwater-Climate or human impact? J. Hydrol. Reg. Stud. 22, 100597 (2019). https://doi. org/10.1016/j.ejrh.2019.100597

Haas, J.C., Birk, S.: Climate change vs. human impact. A look into Austrian groundwater, EGU General Assembly 2020, Online, 4-8 May 2020, EGU2020-8148 (2020) https://doi.org/10.5194/ egusphere-egu2020-8148

Hanauer, B., Möbus, C.: Quantifizierung der Grundwassersströmung in einem alpinen Talkessel. Grundwasser 25(1), 43-52 (2020). https://doi.org/10.1007/s00767-019-00443-w

Harum, T., Dalla-Via, A., Reszler, C.: Grundwassermodell Salzachtal Abschnitt Königseeache: Kuchl Endbericht. Joanneum Research Resources, Bergheim (2016)

Höfer-Öllinger, G.: Untersuchungen der Karsthydrogeologie im Land Salzburg, Österreich. Grundwasser - Zeitschrift der Fachsekti- on Hydrogeologie 25(1), 15-29 (2020). https://doi.org/10.1007/ s00767-019-00439-6

Holman, I.P.: Climate change impacts on groundwater recharge-uncertainty, shortcomings, and the way forward? Hydrogeol J 14(5), 637-647 (2006). https://doi.org/10.1007/s10040-005-0467-0

$\mathrm{Hu}$, L.Y., Chugunova, T.: Multiple-point geostatistics for modeling subsurface heterogeneity: A comprehensive review. Water Resour. Res. 44(11), 1-14 (2008). https://doi.org/10.1029/ 2008WR006993

Huysmans, M., Dassargues, A.: Application of multiple-point geostatistics on modelling groundwater flow and transport in a crossbedded aquifer (Belgium). Hydrogeol J 17(8), 1901-1911 (2009). https://doi.org/10.1007/s10040-009-0495-2

Jandrisevits, C., Marschallinger, R., Hofmann, T.: Multivariate geostatistical analysis of sedimentary infill in the Upper Salzach valley, Austria. Austrian J Earth Sci 107(2), 89-99 (2014)

Kersch, P.: Hochwasserschutz Reischenbach: Technischer Bericht, Projektsteil Grundwasser, GZ 2619. Salzburg, Austria (2016)

Kessler, T.C., Comunian, A., Oriani, F., Renard, P., Nilsson, B., Klint, K.E., Bjerg, P.L.: Modeling fine-scale geological heterogeneityexamples of sand lenses in tills. Groundwater (2013). https://doi. org/10.1111/j.1745-6584.2012.01015.x

Klammler, G., Frank, J., Kupfersberger, H.: Conceptual approach to investigating the impact of climate change scenarios on groundwater recharge, nitrogen leaching and maize yield predictions at Wagna test site, Austria. Bodenkultur 64(1-2), 27-38 (2013)

Kløve, B., Ala-Aho, P., Bertrand, G., Gurdak, J.J., Kupfersberger, H., Kværner, J., Muotka, T., Mykrä, H., Preda, E., Rossi, P., Uvo, C.B., Velasco, E., Pulido-Velazquez, M.: Climate change impacts on groundwater and dependent ecosystems. J. Hydrol. Reg. Stud. 518, 250-266 (2014). https://doi.org/10.1016/j.jhydrol.2013.06. 037

Lasagna, M., Mancini, S., de Luca, D.A.: Groundwater hydrodynamic behaviours based on water table levels to identify natural and anthropic controlling factors in the Piedmont Plain (Italy). Sci. Total Environ. 716, 137051 (2020). https://doi.org/10.1016/j.scitotenv. 2020.137051

Marschallinger, R., Orsi, G., Burger, U., Poscher, G.: Multiple Point Geostatistics for the 3D-modeling of Geological Formations. ISRM Regional Symposium-EUROCK 2015, Salzburg, Austria; International Society for Rock Mechanics and Rock Engineering (Hrsg.) (2014)

Meinshausen, M., Smith, S.J., Calvin, K.: The RCP greenhouse gas concentrations and their extensions from 1765 to 2300. Clim Change 213, 109 (2011). https://doi.org/10.1007/s10584-0110156-Z

Moss, R.H., Edmonds, J.A., Hibbard, K.A., Manning, M.R., Rose, S.K., van Vuuren, D.P., Carter, T.R., Emori, S., Kainuma, M., Kram, T., Meehl, G.A., Mitchell, J.F., Nakicenovic, N., Riahi, K., Smith, S.J., Stouffer, R.J., Thomson, A.M., Weyant, J.P., Wilbanks, T.J.: The next generation of scenarios for climate change research and assessment. Nature 463, 747-756 (2010)

Plöchinger, B.: Salzburger Kalkalpen. Sammlung geologischer Führer, Bd. 73. Borntraeger, Berlin, S. 1-144 (1983)

Plöchinger, B.: Geologische Karte von Hallein. Geologische Karte der Republik Österreich 1:50.000. Geologische Bundesanstalt, Wien, S. 94 (1987)

Pomper, J., Salcher, B.C., Eichkitz, C., Prasicek, G., Lang, A., Lindner, M., Götz, J.: The glacially overdeepened trough of the Salzach Valley, Austria: Bedrock geometry and sedimentary fill of a major Alpine subglacial basin. Geomorphology 295, 147-158 (2017). https://doi.org/10.1016/j.geomorph.2017.07.009

Riahi, K., Rao, S., Krey, V.: RCP 8.5-A scenario of comparatively high greenhouse gas emissions. Clim Change 109, 33 (2011). https://doi.org/10.1007/s10584-011-0149-y

Schälchli, U. (1993) Die Kolmation von Fliessgewässersohlen: Prozesse und Berechnungsgrundlagen (Clogging of River Beds: Proces- 
ses and Theory), Ph.D. thesis, ETH Diss. Nr. 10293, Swiss Fed. Inst. of Technol., Zurich, Switzerland (1993)

Scibek, J., Allen, D.M.: Modeled impacts of predicted climate change on recharge and groundwater levels. Water Resour. Res. (2006). https://doi.org/10.1029/2005WR004742

Seefeldner, E.: Entstehung und Alter der Salzburger Ebene. Mitteilungen der Gesellschaft für Salzburger Landeskunde, Bd. 94., S. 202-208 (1954)

Stocker, T.F., Qin, D., Plattner, G.-K., Tignor, M., Allen, S.K., Boschung, J., Nauels, A., Xia, Y., Bex, V., Midgley, P.M. (Hrsg.): IPCC: Climate Change 2013: The Physical Science Basis. Contribution of Working Group I to the Fifth Assessment Report of the Intergovernmental Panel on Climate Change. Cambridge University Press, Cambridge, United Kingdom, New York, S. 1-1535 (2013) https://doi.org/10.1017/CBO9781107415324

Strebelle, S.: Conditional simulation of complex geological structures using multiple-point statistics. Math Geol 34(1), 1-21 (2002)

Stummer, E.: Der Aufbau des Salzburger Zungenbeckens. Mitteilungen der Gesellschaft für Salzburger Landeskunde, Bd. 86-87., S. 91-92 (1947)

Tague, C., Grant, G.E.: Groundwater dynamics mediate low-flow response to global warming in snow-dominated alpine regions. Water Resour. Res. 45(7), 181 (2009). https://doi.org/10.1029/ 2008WR007179

Taylor, R.G., Scanlon, B., Döll, P., Rodell, M., van Beek, R., Wada, Y., Longuevergne, L., Leblanc, M., Famiglietti, J.S., Edmunds,
M., Konikow, L., Green, T.R., Chen, J., Taniguchi, M., Bierkens, M.F.P., MacDonald, A., Fan, Y., Maxwell, R.M., Yechieli, Y., Gurdak, J.J., Allen, D.M., Shamsudduha, M., Hiscock, K., Yeh, P., Holman, I., Treidel, H.: Ground water and climate change. Nature Clim Change 3(4), 322-329 (2013). https://doi.org/10. 1038/nclimate1744

Van Husen, D.: Verbreitung, Ursachen und Füllung glazial übertiefter Talabschnitte an Beispielen in den Ostalpen. Eiszeitalt. Ggw 29, 9-22 (1979)

Wessely, G., Neubauer, F., Salcher, B., Wagreich, M.: A geological snapshot from the front of the Northern Calcareous Alps: Well Obermoos TH-1, Salzburg, Austria. Austrian. J. Earth. Sci. 109(2), 189-202 (2016). https://www.univie.ac.at/ajes/archive/ volume_109_2/wessely_et_al_ajes_109_2.pdf

ZAMG - Zentralanstalt für Meteorologie und Geodynamik: Download Niederschlagszeitreihen für die Messstellen „Salzburg Freisaal“ und „Salzburg Flughafen“ von der Webseite (2019). https:// www.zamg.ac.at/cms/de/produkte/klima/daten-und-statistiken/ messdaten, Zugegriffen: 17. Okt. 2019

Hinweis des Verlags Der Verlag bleibt in Hinblick auf geografische Zuordnungen und Gebietsbezeichnungen in veröffentlichten Karten und Institutsadressen neutral. 